
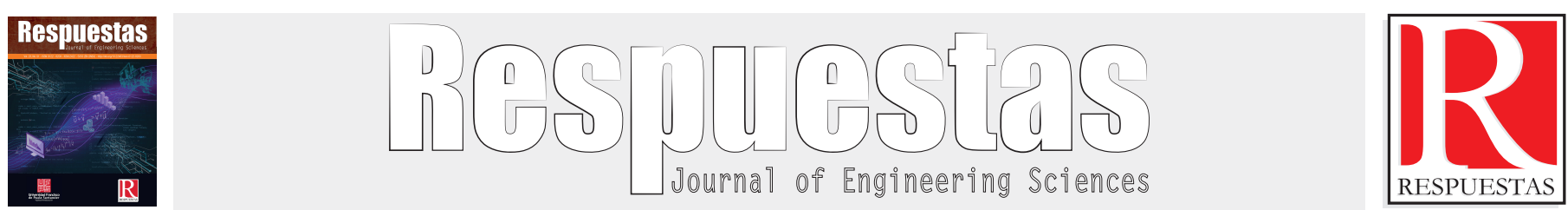

Artículo Original

https://doi.org/10.22463/0122820X.1528

\title{
El esfuerzo físico y la salud en actividades recreativas y deportivas
}

Physical exertion and health in recreational and sporting activities

Rafael Enrique Lozano-Zapata, Yusbely Cecilia Castrillón-Jaimes, María Susana Peña-Reyes, Brian Johan Bustos-Viviescas.

${ }^{a}$ Doctor en Ciencias de la Actividad Física y el Deporte, rafaenloza@unipamplona.edu.co, orcid.org/0000-0002-6239-5883, Universidad de Pamplona, Cúcuta, Colombia.

${ }^{b}$ Magister en Práctica Pedagógica. yusbely.castrillon@unipamplona.edu.co, orcid.org/0000-0003-4205-4018, Universidad de Pamplona, Cúcuta, Colombia.

${ }^{c}$ Licenciada en Pedagogia Infantil (c), maria.pena@unipamplona.edu.co, orcid.org/0000-0002-3280-8552, Universidad de Pamplona, Cúcuta, Colombia.

${ }^{d}$ Licenciado en Educación Básica con Énfasis en Educación Física, Recreación y Deportes (c), brian.bustos@unipamplona.edu.co, orcid.org/0000-0002-4720-9018, Universidad de Pamplona, Cúcuta, Colombia.

Cómo citar: R.E. Lozano-Zapata, Y.C. Castrillón-Jaimes, M.S. Peña-Reyes y B.J. Bustos-Viviescas, "El esfuerzo físico y la salud en actividades recreativas y deportivas", Respuestas, vol. 23, no. S1, pp. 105 - 108, 2018. https://doi.org/10.22463/0122820X.1528

Recibido: Enero 29, 2018; Aprobado: Junio 28, 2018

\begin{tabular}{|c|c|}
\hline & RESUMEN \\
\hline Palabras Clave: & $\begin{array}{l}\text { Antecedentes: El esfuerzo que se produce por la actividad física trae consigo múltiples beneficios, pero realizarlo de una forma } \\
\text { programada, sistemática y con controles periódicos, se puede lograr observar los cambios que genera el ejercicio }\end{array}$ \\
\hline Actividad Física & fisiológicamente, en pro de la salud y la condición física; cada fin de semana el ejercitarse en los espacios que ofrece la ciudad, \\
\hline Condición & la cantidad de personas y las diferentes actividades que se promueven para realizar ejercicio, deporte, arte y otras actividades \\
\hline Esfuerzo & puede que no conlleven a realizar el esfuerzo físico requerido para mantener una óptima condición física. Objetivos: por este \\
\hline Salud & $\begin{array}{l}\text { motivo el estudio se enfoca en reconocer las necesidades de esta población que asiste a la ciclovía cada semana y poder orientar } \\
\text { de una forma más apropiada a quienes no conocen de qué manera realizar una actividad física, en pro de su salud. Métodos: El } \\
\text { estudio presentó un enfoque mixto de tipo descriptivo donde se miden variables independientes el cual tuvo un diseño de campo } \\
\text { para conocer los beneficios que trae realizar una fuerza y el ejercitarse como buena condición física y de salud. Resultados: para } \\
\text { el logro de este objetivo se realizó una encuesta estructurada en la cual participaron } 222 \text { hombres ( } 30,98 \pm 14,24 \text { años) y } 234 \\
\text { mujeres ( } 28,47 \pm 12,24 \text { años), además se aplicó el Test de Ruffier-Dickson (TRD) para el cuál se tomó la frecuencia cardiaca en } \\
\text { reposo, al final y recuperación a } 181 \text { personas de las cuales } 143 \text { realizan actividad física regularmente (Grupo AF) y } 38 \text { personas } \\
\text { que no realizaban actividad física regularmente (Grupo NAF). Conclusiones: la muestra participante presentaba deficiencias en } \\
\text { algunos aspectos valorados, así mismo en la recolección de las valoraciones el beneficio del grupo AF presentó un IMC y \% G } \\
\text { menor que el grupo NAF puesto que en los demás datos ambos grupos obtuvieron valores muy similares. }\end{array}$ \\
\hline
\end{tabular}

\section{ABSTRACT}

\section{Keywords:}

Physical Activity

Condition

Effort

Health
Background: The effort that is produced by the physical activity brings with it multiple benefits, but to realize it of a programmed form, systematic and with periodic controls, it is possible to be able to be observed the changes that generates the exercise physiologically, in pro of the health and the physical condition; every weekend the exercise in the spaces that offers the city, the quantity of people and the different activities that are promoted to realize exercise, sport, art and other activities can that they do not take to realize the physical effort required to maintain an optimal physical condition. Objectives: for this reason the study focuses on recognizing the needs of this population that attends the ciclovía every week and to be able to orient in a more appropriate way to those who do not know how to carry out a physical activity, in favor of their health. Methods: The study presented a mixed approach of descriptive type where independent variables are measured, which had a field design to know the benefits of strength and exercise as good physical condition and health. Results: in order to achieve this objective, a structured survey was carried out in which 222 men ( $30.98 \pm 14.24$ years) and 234 women ( $28.47 \pm 12.24$ years) participated. In addition, the Ruffier-Dickson Test (TRD) was applied, for which the heart rate was taken at rest, at the end and recovery to 181 people of whom 143 carry out physical activity regularly (AF Group) and 38 people who did not carry out physical activity regularly (NAF Group). Conclusions: the participating sample presented deficiencies in some aspects valued, as well as in the collection of valuations the benefit of the AF group presented a BMI and \% G lower than the NAF group since in the other data both groups obtained very similar values.

\section{Introducción}

La actividad física es fundamental practicarla para tener no solamente un buen estado físico, sino también una buena salud, sin embargo es importante saber practicarla regularmente y de una forma programa, teniendo en cuenta el esfuerzo físico, la capacidad del individuo, el tiempo apropiado y la intensidad adecuada en el momento de realizarla, así lograr tener un beneficios considerables y no generar ningún tipo de situación adversa, como puede ser dolencias musculares, lesiones deportivas o patologías, las cuales perjudican la salud del sujeto [1]. De esta manera la calidad de vida, es decir, la posibilidad de que las personas y los grupos desarrollen sus potencialidades, se convierte desde esta concepción orientada al bienestar en el referente cualitativo de la actividad física relacionada con la salud [2].

El cuerpo del ser humano está preparado para moverse, más aún, tiene la necesidad de hacerlo. No obstante, a pesar de esta necesidad, la práctica de actividad física en la población es cada vez menos frecuente, muy especialmente en niños, esto debido a el desarrollo tecnológico [3]. Estos bajos niveles de práctica de actividad física conllevan el sedentarismo de buena parte de la población, lo que unido a unos hábitos alimentarios nada idóneos han provocado el aumento alarmante de diferen-

*Autor de correspondencia. 
tes patologías (obesidad, enfermedades cardiovasculares, síndrome metabólico etc...) que, en algunos casos, se han convertido en un auténtico problema de Salud Pública, para cada nación [4].

Por lo tanto, si el esfuerzo que se realiza en un ejercicio físico está bien hecho, este contribuye a disminuir los efectos de lesiones proporcionando beneficios en diferentes funciones. No existen límites de edad para los beneficios del ejercicio, y entre esos se pueden mencionar el incremento del tono y la masa muscular, y por tanto la fuerza; la mejoría de las condiciones hemodinámicas y de la mecánica ventilatoria y su eficiencia; la disminución de la tensión arterial; la prevención de la arteriosclerosis, la hiperlipidemia y la osteoporosis; la ayuda en el control de la diabetes; así como la mejoría en la flexibilidad, el equilibrio y la movilidad articular [5].

De esta manera es importante controlar y evaluar la actividad física que se lleve a cabo, con el fin de observar las adaptaciones fisiológicas que genera y todos los beneficios de la misma [1], [6]. Para conocer estas condiciones se tienen diferentes instrumentos como lo son cuestionarios avalados internacionalmente, pero se consideran que son de una forma subjetiva, ya que no se puede cuantificar con exactitud lo expresado por los participantes, no obstante para tener un evaluación de la condición física y de salud más real de los sujetos con mayor objetividad y precisión, encontramos test de laboratorio o de campo, con equipos de alta tecnología, donde sea más real y actual estos resultados, para que con base a estos se pueda llevar a cabo un plan o programa de adaptación física más apropiado y el cual genere beneficios para la salud [1],[7].

Teniendo en cuenta estos aspectos expresados en la literatura actual, a través de esta investigación se planteó intervenir en la población asistente a la ciclovía de los días domingos en la ciudad de Cúcuta; con el fin conocer que las personas asistentes a la ciclovía realizan actividad física de una forma regular y quienes no; de igual manera conocer si las personas que realizan la actividad física, la controlan de una forma precisa y adecuada, para el beneficio de la misma y sino por ende le genera algún tipo de malestar, para luego orientar a quienes no realizan la actividad física apropiadamente con base a los resultados obtenidos en las variables controladas en el chequeo previo a realizar el ejercicio en la ciclovía.

\section{Materiales y métodos}

El presente estudio presento un enfoque mixto de tipo descriptivo de medición de variables independientes el cual tuvo un diseño de campo para conocer los beneficios que trae realizar un esfuerzo y actividad física para conseguir un buen estado físico y salud de las personas que asisten los días domingos a la ciclovía de la ciudad de Cúcuta.
Participaron 456 personas que asistieron a la ciclovía, las cuales de una forma voluntaria se acercaron a nuestro stand de la Universidad de Pamplona, donde se les explicó cuál eran los propósitos del estudio; seguidamente firmaron un consentimiento informado por escrito, donde nos autorizaron a intervenir y poder manipular los resultados con fines investigativos, siempre respetando la confidencialidad de los participantes. De igual manera se tuvieron en cuenta los principios de la declaración de Helsinki y los principios para investigaciones en ciencias del deporte.

El análisis estadístico se realizó en PSPP para Windows 7, en este software se introdujeron los resultados y se tuvo en cuenta los resultados descriptivos de cada una de las variables, tanto valores medios como desviación estándar.

De acuerdo a las personas que asistieron a las valoraciones realizadas en la ciclovía, participaron 222 hombres $(30,98 \pm 14,24$ años) y 234 mujeres $(28,47 \pm 12,24$ años). Teniendo como características una masa corporal total promedio de los sujetos evaluados de género masculino de $72,73 \pm 13,87 \mathrm{~kg}$; una edad promedio de $34,40 \pm 16,62$ años y una talla de 170,79 $\pm 7,78 \mathrm{~cm}$. Para las mujeres se encontraron las siguientes características una masa corporal total promedio de 58,78 \pm $10,42 \mathrm{~kg}$; una edad promedio de $30,38 \pm 15,76$ años y una talla en promedio de $160,48 \pm 6,96 \mathrm{~cm}$.

También se obtuvo información con la aplicación del cuestionario estructurado, respondiendo de forma voluntaria cada pregunta para conocer si al hacer ejercicio realizan fuerza o si lo hacen de manera apropiada o quienes no, al igual si se llevaba a cabo un calentamiento adecuado o que tanto se hidrataban durante la actividad física.

Seguidamente las valoraciones que se determinaron arrojaron el índice de masa corporal (IMC), presión arterial, frecuencia cardiaca en reposo, dinamometría de mano, porcentaje graso y el test de Ruffier-Dickson, para así conocer la capacidad de cada sujeto y de acuerdo a los datos adquiridos sugerir unas orientaciones apropiadas al momento de realizar la actividad física.

\section{Resultados y análisis}

Teniendo en cuenta todo lo expuesto en la metodología y de acuerdo al cuestionario estructurado aplicado a los individuos participantes en el estudio, quienes asisten de forma regular a la ciclovía los días domingos en la ciudad de Cúcuta. Se encontraron las siguientes respuestas y las más relevantes, de acuerdo a lo preguntado.

345 personas si realizan actividad física regularmente $(75,66 \%), 272$ personas no realizan su actividad física solamente en el malecón los días domingo (59,65\%), la 
frecuencia promedio de entrenamiento fue de $3 \pm 2,39$ sesiones por semana y con una duración promedio de la sesión de $1,23 \pm 0,96$ horas, 158 personas respondieron que no realizan calentamiento previo a la actividad física $(39,45 \%), 181$ personas respondieron no tener conocimientos sobre la actividad física que desarrollaban en el malecón (39,69\%), 334 personas respondieron que no controlaban sus variables fisiológicas (FC, VO2 max., entre otros) (76,25\%), 180 personas respondieron no tener hábitos alimenticios adecuados $(39,47 \%), 93$ personas respondieron que se hidrataban adecuadamente (20,39\%), 270 personas respondieron que no han realizado chequeos fisiológicos para conocer su condición física y salud $(59,21 \%), 449$ personas respondieron que si desearían controlar su avance y adaptación de la condición física por medio de variables fisiológicas $(98,46 \%)$, entre las actividades físicas preferenciales por los encuestados se encuentran principalmente el caminar $(30,04 \%)$, trotar $(23,68 \%)$, gimnasio $(12,5 \%)$ y andar bicicleta $(10,96 \%)$.

En el momento de realizar las diferentes valoraciones antes de realizar algún tipo de actividad física, se tomaron las variables de frecuencia cardiaca en reposo, fuerza en mano, presión arterial, IMC y porcentaje graso, donde se han diferenciado las variables tanto para hombres como mujeres, encontrando los resultados en la tabla I.

Tabla I. Variables fisiológicas controladas antes de iniciar la actividad física en la ciclovía

\begin{tabular}{|c|c|c|}
\hline Variable & Hombres $(\mathbf{n = 2 2 2})$ & Mujeres $(\mathbf{n = 2 3 4})$ \\
\hline $\begin{array}{c}\text { Frecuencia Cardiaca } \\
\text { Reposo }(\mathrm{ppm})\end{array}$ & $82,33 \pm 15,66$ & $85,86 \pm 4,35$ \\
\hline IMC & $24,53 \pm 4,37$ & $23,04 \pm 4,03$ \\
\hline $\begin{array}{c}\text { Dinamometría Mano } \\
\text { Derecha }(\mathrm{kg})\end{array}$ & $36,78 \pm 9,59$ & $24,81 \pm 9,33$ \\
\hline $\begin{array}{c}\text { Dinamometría Mano } \\
\text { Izquierda (kg) }\end{array}$ & $35,71 \pm 9,71$ & $23,33 \pm 5,91$ \\
\hline Presión Arterial (mmHg) & $\begin{array}{c}116,15 / 76,72 \pm \\
19,28 / 48,21\end{array}$ & $\begin{array}{c}107,88 / 69,62 \pm \\
18,77 / 14,56\end{array}$ \\
\hline \% Graso & $19,25 \pm 8,74$ & $25,81 \pm 7,22$ \\
\hline
\end{tabular}

Y por último, se llevó a cabo el test de Ruffier-Dickson, con el fin de conocer la condición física de los evaluados durante la presente investigación, de esta manera orientar la actividad física de una forma apropiada, de las personas que asisten a la ciclovía y no sabe ejecutar la actividad física apropiadamente.

Teniendo en cuenta la clasificación de este test y la literatura actual, se encontró que los hombres tienen un valor medio de este índice de $12,27 \pm 5,45$, donde están en un nivel INAPTO, y para las mujeres evaluadas durante la presente investigación encontramos como valor medio en esta variable de 12,79 \pm 5,40 , estando también en un valor INAPTO, de acuerdo a la clasificación, pero se debe tener en cuenta que la mayoría de estas personas van de domingo a domingo a realizar actividad física y muy pocos realizan actividad física durante la semana, de una forma apropiada.

Como se ha venido manifestando en diferentes estudios, a mayor actividad física, no solamente se mejora la condición física, sino también se disminuye la capacidad de adquirir enfermedades crónicas no transmisibles, siempre y cuando se realice una fuerza, un movimiento de manera adecuada. A nivel mundial ha aumentado el número de personas que no realizan actividad física de una forma programa y adecuada, esta inactividad influenciada por el trabajo, la tecnología y falta de interés [1], [3], [4], [7].

El fin de conocer diferentes variables con los asistentes a la ciclovía el día domingo, donde se logró conocer las diferentes valoraciones dadas para el desarrollo de la actividad física se encontró que la mayoría de ellos hacen actividad física, un $75,66 \%$ de todas las personas participantes y el restante no hace regularmente, pero lo curioso es que la mayoría de ellos no conocen lo que realizan, es decir de una forma programa y planificada, al igual no realizan un calentamiento adecuado y menos desarrollan una recuperación finalizado el esfuerzo físico. Esto influye negativamente para mejorar el estado físico y la salud de las personas [1], [8].

En el momento de analizar las variables pre-esfuerzo, se encuentra que las personas tienen una frecuencia cardiaca en reposo apropiada, de acuerdo a toda la población en general, la presión arterial tiene valores adecuado de acuerdo a personas aparentemente sanas, teniendo un valor adecuado. $(116,15 / 76,72 \pm 19,28 / 48,21 \mathrm{mmHg})$, para hombres y $(107,88 / 69,62 \pm 18,77 / 14,56 \mathrm{mmHg})$ para mujeres. De esta manera se observan valores similares a otros estudios y con personas en similares condiciones [9], [10].

En cuanto al IMC y el porcentaje graso, se observa que teniendo en cuenta los resultados encontrados tanto para hombres como para mujeres se encuentran en un normal peso (aceptable) de acuerdo a diferentes estudios y tablas comparativas, encontrando valores para hombres de $24,53 \pm 4,37$ y para mujeres de 23,04 $\pm 4,03$. De acuerdo al porcentaje graso se observa que los hombres su valor medio es de 19,25 $\pm 8,74 \%$, encontrándose en un valor promedio para la población y en cuanto a las mujeres se observa un valor de $25,81 \pm 7,22 \%$, donde se clasifican en un valor sobrepeso, estando más elevado en las mujeres en relación a los hombres [11], [12].

Con respecto a la condición física determinada a través del test de Ruffier-Dickson, tanto hombres como mujeres se encontraron resultados no favorables, encontrando en un valor no apto, elevado de acuerdo a la clasificación, de tal manera que se observa con otros estudios que estos datos son elevados en relación a otros sujetos de igualdad de condiciones [13]. 


\section{Conclusiones}

Teniendo en cuenta los resultados obtenidos y la descripción de las variables, se ha logrado definir un perfil descriptivo de los asistentes a la ciclovía los días domingos en la ciudad de Cúcuta, encontrando que a pesar que las personas participantes en la presente investigación la mayoría de ellos no hacen actividad física de una forma regular y apropiada, donde no se controlan las variables fisiológicas, pese a ello, estos resultados son favorables para los individuos participantes, donde cabe resaltar que se debe realizar previo calentamiento muscular y articular para así prevenir lesiones y al finalizar el ejercicio físico realizar un proceso de recuperación adecuado. Esto generará una mejor condición física en los adultos mayores y también optimizará la salud, para prevenir enfermedades crónicas no transmisibles.

\section{Referencias}

[1] R. Lozano-Zapata, "La actividad física, como factor fundamental del buen estado físico y salud de las personas que asisten los días domingos a la ciclovía del malecón de la ciudad de Cúcuta", Memorias III Semana Internacional, XI Semana de Ciencias, Tecnología e Innovación. 2016, pp. 409-413.

[2] V. Pérez-Samaniego y J. Devis-Devis, "La promoción de la actividad física relacionada con la salud. La perspectiva de proceso y de resultado", Revista Internacional de Medicina y Ciencias de la Actividad Física y el Deporte, vol. 3, no. 10, pp. 69-74, 2003.

[3] J. Duperly y F. Lobelo, (2015). Prescripción del Ejercicio, una guía para recomendar actividad física a cada paciente. Bogotá: Ediciones de la U, 2015, pp. 19-77.

[4] Y. Escalante, "Actividad física, ejercicio físico y condición física en el ámbito de la salud pública”, Revista Española Salud Pública, vol. 85, no. 4, pp. 325-328, 2011.

[5] O. Cintra y Y. Balboa, "La actividad física: un aporte para la salud", Lecturas: Educación Física y Deportes, Año 16, no. 159, 2011, [En línea]. Disponible en: http://www.efdeportes.com/efd159/la-actividad-fisica-para-la-salud.htm. [Accedido:15-agos-2017]

[6] W. Ramírez, S. Vinaccia y G. Ramón Suárez, "El impacto de la actividad física y el deporte sobre la salud, la cognición, la socialización y el rendimiento académico: una revisión teórica", Revista de Estudios Sociales, no. 18, pp. 67-75, 2004.

[7] O. Rubiano, Prescripción del ejercicio en personas aparentemente sanas. Bogotá: Editorial Kinesis, 2013.

[8] G. Ortega, A. Cano y L. Palma, "Actividad Física y Salud",
I Congreso Internacional en Investigación y Didáctica de la Educación Física. II Jornadas de Investigadores en Didáctica de la Educación Física. 2016, pp.246-249.

[9] A. Gómez-Cabello, et al., "Organized physical activity in the elderly, a tool to improve physical fitness during ageing", Revista Española de Salud Pública, vol. 92, 2018, pii: e201803013.

[10] C.R. Pedrero, et al., "Higher levels of physical fitness are associated with a reduced risk of suffering sarcopenic obesity and better perceived health among the elderly: the EXERNET multi-center study", The journal of nutrition, health \& aging, vol. 19, no. 2, pp. 211-217, 2015.

[11] C. Geisler, L. Schweitzer y M.J. Muller, "Functional correlates of detailed body composition in healthy elderly subjects, Journal of Applied Physiology, vol. 124, no. 1, 2018.

[12] F. Sartor, et al., "A. 45-Second Self-Test for Cardiorespiratory Fitness: Heart Rate-Based Estimation in Healthy Individuals", PLoS One, vol. 1, no. 12, pp. 1-12, 2016.

[13] R. Latorre, J. Salas-Sánchez y V.M. Soto-Hermoso, "Composición corporal relacionada con la salud en atletas veteranos", Revista Nutrición Hospitalaria, vol. 27, no. 4, pp. 1236-1246, 2012. 